\section{rev Psi}

Revista de Psicología (UNLP)

https://revistas.unlp.edu.ar/revpsi

Artículo de investigación

\title{
Síndrome de Münchausen por poderes. Alto riesgo para la vida de niños, niñas y adolescentes
}

\section{Graciela Susana Lovaglio ${ }^{1}$}

Correspondencia

liclovaglio@hotmail.com

Filiaciones institucionales

${ }^{1}$ Hospital General de Niños Dr. Pedro de Elizalde (Argentina)
Patricia Del Bagge ${ }^{1}$

\section{Resumen}

El presente artículo tuvo como objetivo realizar una revisión del síndrome de Münchausen por poderes para contribuir con el diagnóstico precoz de este tipo de maltrato infantil de alto riesgo. Una de las metas fue difundirlo entre los profesionales de los equipos de salud, los organismos de protección de derechos de niños/as y adolescentes, la justicia y las instituciones escolares. Se describió el recorrido histórico del concepto y se abordaron sus características, manifestaciones clínicas e indicadores. Fue presentado un modelo inédito de relevamiento de datos para efectores de salud. Se elaboró un registro epidemiológico con datos obtenidos de la Unidad de Violencia Familiar del Hospital de Niños Pedro de Elizalde, del período 2001-2017, cuyo resultado fue de 24 casos confirmados. Fue descripta la estrategia diagnóstica durante la internación de niños/as y adolescentes, el comportamiento del perpetrador, los aspectos contra-transferenciales del equipo tratante, el egreso y el tratamiento psicológico del niño.

\section{Palabras clave}

síndrome de Münchausen por poderes | maltrato infantil | perpetrador | diagnóstico temprano | internación pediátrica

\section{Cómo citar}

Lovaglio, G. S. y Del Bagge, P. (2021). Síndrome de Münchausen por poderes. Alto riesgo para la vida de niños, niñas y adolescentes. Revista de Psicología, 20(2), 123-138. HTTPS://DX.DOI. ORG/10.24215/2422572XEO82

\section{Proceso editorial}

Recibido

6 jul. 2020

Aceptado

2 feb. 2021
1 ra decisión

12 dic. 2020

Publicado 15 feb. 2021
ISSN

2422-572X

Licencia

Licencia de Cultura Libre CC-BY 4.0

(Compartir - Adaptar - Atribuir)

Entidad editora

RevPsi es una publicación de la

Facultad de Psicología (Universidad

Nacional de La Plata, Argentina) 


\section{Sindrome de Munchausen por procuraçā. Alto risco para a vida de crianças e adolescentes}

\section{Resumo}

O objetivo deste artigo foi realizar uma revisão da síndrome de Münchausen por procuração, a fim de contribuir para o diagnóstico precoce desse tipo de abuso infantil de alto risco. Um dos objetivos era divulgá-lo entre os profissionais das equipes de saúde, as entidades de defesa dos direitos da criança e do adolescente, a justiça e as instituições escolares. Descreveu-se a trajetó-ria histórica do conceito e abordou-se suas características, manifestações clínicas e indicadores. Um modelo de coleta de dados sem precedentes para prestadores de cuidados de saúde foi apresentado. Foi elaborado um registro epidemiológico com dados obtidos na Unidade de Vio-lência Familiar do Hospital Infantil Pedro de Elizalde, no período de 2001 a 2017, cujo resulta-do foi de 24 casos confirmados. Descreveu-se a estratégia diagnóstica durante a internação de crianças e adolescentes, o comportamento do agressor, os aspectos contratransferenciais da equipe de atendimento, a alta hospitalar e o tratamento psicológico da criança.

\section{Palavras-chave}

síndrome de Münchausen por poder | maltrato infantil | autor | diagnóstico precoce | hospitaliza-ção pediátrica

\section{Anxiety in the Argentine population in the context of the CO- VID-19 pandemic}

\section{Abstract}

The objective of this article was to carry out a review of the Münchausen syndrome by proxy to contribute to the early diagnosis of this type of high-risk child abuse. One of the goals was to disseminate it among the professionals of the health teams, the organizations that protect the rights of children and adolescents, the justice system and school institutions. The historical journey of the concept was described and its characteristics, clinical manifestations and indicators were addressed. An unpublished data collection model for health care providers was presented. An epidemiological registry was prepared with data obtained from the Family Violence Unit of the Pedro de Elizalde Children's Hospital, from the period 2001-2017, the result of which was 24 confirmed cases. The diagnostic strategy during the hospitalization of children and adolescents, the behavior of the perpetrator, the counter-transference aspects of the treating team, the discharge and the psychological treatment of the child were described.

\section{Keywords}

Munchausen syndrome | child abuse | perpetrator | early diagnosis | hospitalization 


\section{Aspectos destacados del trabajo}

- Desmitificación de creencias sobre la capacidad innata de las madres para prodigar cuidados.

- Al llegar a la pubertad muchos niños y niñas participan del maltrato pudiendo ejercerlo sobre otros a futuro.

- Las características de personalidad de las madres perpetradoras refuerzan la letalidad del síndrome.

- La exploración de datos ocultos y los antecedentes del niño facilitan el diagnóstico.

El término síndrome de Münchausen es utilizado por Asher en 1951 (como se citó en Indart, 2018) para referirse al cuadro en el que un adulto fabrica síntomas caracterizados por el abuso de consultas hospitalarias, a raíz de significativos padecimientos que promueven exploraciones, en ocasiones invasivas y tratamientos médicos innecesarios. Lo denomina así con referencia al Barón Karl Friederich Hieronymus Von Münchausen, alemán del siglo XVI, que relataba fantásticas historias de sus proezas.

El pediatra británico Meadow, le da el nombre de síndrome de Münchausen por poder o por delegación al trastorno facticio por el cual la enfermedad del niño es sugerida, inducida o fabricada por la persona más próxima a él, en general la madre. Plantea esta denominación a partir de dos casos clínicos, en uno de ellos la madre manifiesta que el niño presenta hematuria, pero más tarde se comprueba que la orina había sido contaminada con sangre. En el otro, el niño había sido intoxicado con cloruro de sodio. En ambos casos, los pacientes habían ingresado a diversos centros de salud y habían sido sometidos a múltiples evaluaciones médicas, sin arribar a ningún diagnóstico comprobable (Meadow, 2002, como se citó en Garrote et al., 2008).

Verity (1979, como se citó en Indart, 2018) define como síndrome de Polle, al cuadro clínico simulado por los padres a través de supuestos síntomas que presentaban sus hijos. Se lo llamó Polle en honor al hijo del Barón Münchausen, quien muriera a los diez meses por una causa misteriosa. Esta denominación es controvertida y no empleada, por no haberse comprobado la culpabilidad de sus padres.

La Asociación Americana de Psiquiatría ([AAP], 1994), en el Manual diagnóstico y estadístico de los trastornos mentales (DSM-IV), lo define como trastorno facticio no especificado. En esta clasificación incluye la denominación trastorno facticio por poderes, en el apéndice de criterios y ejes propuestos para estudios posteriores.

El DSM-IV TR (AAP, 2002) lo denomina desorden facticio por poderes y en la 
actualidad, el DSM-5 (AAP, 2014) trastorno facticio aplicado a otro, incluyéndolo dentro de trastorno facticio en la categoría de trastornos de síntomas somáticos y trastornos relacionados.

La última denominación es la de Glaser y Davis (2019), quien lo menciona como enfermedad inducida o producida por cuidadores.

\section{Características y manifestaciones clínicas}

En el abordaje del síndrome de Münchausen por poderes (SMPP), intervienen aspectos médicos, psicológicos, psiquiátricos y legales que atañen tanto al niño como a su familia, los cuales reafirman que para arribar a un diagnóstico es imprescindible una articulación multidisciplinaria.

En relación con el perpetrador, Meadow (2002) refiere que la inductora es la madre biológica en el $98 \%$ de los casos y que existe una coalición con el padre u otro familiar en el 2\% restante. Catalina y Mardomingo (como se citó en Rodríguez Cely et al., 2003) manifiestan que, según sus estudios, el $88,5 \%$ de los casos el autor es la madre, el 2,8\% el padre y el 5,7\% ambos progenitores.

En el diagnóstico de este síndrome, las enfermedades más reportadas involucran a diversos órganos, lo que promueve la interconsulta médico-pediátrica y la indicación de estudios, en ocasiones invasivos. Meadow (1993) refiere que algunos síntomas también se expresan en cambios comportamentales y emocionales, tales como déficit de atención, dificultades en el aprendizaje, trastornos disociativos y psicosis, todos inventados e inducidos por los cuidadores, que en ocasiones han llegado a realizar denuncias de abuso sexual infantil. Estas condiciones van acompañadas del aislamiento social del niño/a y de ausentismo escolar.

En muchos casos, se detectan en los exámenes de laboratorio realizados al paciente, productos químicos o fármacos sin indicación profesional y que la madre refiere nunca haberle suministrado. Los estudios expresan que el etanol, los laxantes y las benzodiacepinas forman parte de los fármacos más comúnmente encontrados.

Estudios realizados describen que la gama de expresiones clínicas es amplia y las presentaciones más comunes son: sangrados, convulsiones, depresión del sistema nervioso central, apneas, diarrea, vómitos, fiebre y erupción cutánea. En menor proporción, los cuadros renales y urológicos, entre los que se encuentran las infecciones urinarias y hematuria (Rosenberg, 1987, Schreier y Libow, 1993, como se citó en Jiménez Hernández, 2015).

Existen nuevas formas de presentación del síndrome de Münchausen por poderes que incluyen a la celiaquía, la deshidratación hipernatrémica, la inmunodeficiencia y la enfermedad de Gaucher.

Meadow (1993) describe en un estudio realizado sobre 14 niños la existencia de falsas denuncias de abuso sexual, fuera del contexto de divorcio, de tenencia o de disputas entre los padres. En seis de los casos, se comprueba que las madres habían 
Tabla 1. Signos y síntomas que se presentan según sistema involucrado y/o diagnóstico médico

\begin{tabular}{|c|c|}
\hline Aparatos y sistemas & Signos y síntomas \\
\hline Alérgico & Alergia alimentaria, exantema. \\
\hline Dermatológico & $\begin{array}{r}\text { Eritema. Vesículas por quemaduras, laceraciones, rasguños, heridas } \\
\text { punzantes, eczema }\end{array}$ \\
\hline Neurodesarrollo & $\begin{array}{r}\text { Trastornos del aprendizaje, déficit de atención/hiperactividad, } \\
\text { disfunciones neuromotoras, retraso generalizado del desarrollo, } \\
\text { psicosis. }\end{array}$ \\
\hline Endócrino & Poliuria, polidipsia, hipoglucemia, diabetes, glucosuria \\
\hline Gastrointestinal & $\begin{array}{r}\text { Dolor abdominal, anorexia, diarrea, deshidratación, quemaduras } \\
\text { esofágicas, vómitos, pérdida de peso, obstrucción intestinal, } \\
\text { disquinesias intestinales, hemorragia, incluyendo hematemesis y } \\
\text { hematoquecía o melena, sangrado por tubo nasogástrico, sangrado } \\
\text { por ileostomía, trastornos que conducen a una necesidad de } \\
\text { administración de nutrición parenteral }\end{array}$ \\
\hline Hematológico & Sangrado, hematomas frecuentes, anemia. \\
\hline Infeccioso & $\begin{array}{r}\text { Fiebre, leucopenia, sepsis, artritis séptica, osteomielitis, falta } \\
\text { de resolución de infecciones con antibióticos para que los que } \\
\text { las bacterias son susceptibles; aparición de nuevas infecciones, } \\
\text { mientras que el niño está recibiendo antibióticos a los que las } \\
\text { bacterias son susceptibles; bacterias inusuales desde el sitio de } \\
\text { infección o infección con múltiples organismos simultáneos de baja } \\
\text { patogenicidad. }\end{array}$ \\
\hline Metabólico & Trastornos mitocondriales, sin pruebas positivas. \\
\hline Neurológico & Convulsiones, cefaleas, mareos, trastornos de la conciencia \\
\hline Oncológico & Leucemia y otros tipos de cáncer. \\
\hline Oftalmológico & $\begin{array}{r}\text { Conjuntivitis hemorrágica, queratitis, edema de párpado, pupilas } \\
\text { desiguales, nistagmo, celulitis periorbitaria }\end{array}$ \\
\hline Ortopédico & Fracturas, esguinces, traumatismos. \\
\hline Ótico & Otorrea, infecciones recurrentes. \\
\hline Renal & $\begin{array}{r}\text { Hematuria, proteinuria, cálculos renales, bacteriuria, insuficiencia } \\
\text { renal, hipertensión, nocturia, hipematremia, hiponatremia, } \\
\text { hipokalemia, piuria, falla renal }\end{array}$ \\
\hline Respiratorio & $\begin{array}{l}\text { Presentación con un evento agudo con peligro de vida, apnea, } \\
\text { incluyendo apnea del sueño, fibrosis quística, sangrado del tracto } \\
\text { respiratorio superior, asma intratable, hemoptisis, cianosis, hipoxia }\end{array}$ \\
\hline Reumatológico & Artritis, artralgia, rigidez matutina. \\
\hline
\end{tabular}

enseñado y alentado el relato a sus hijos. Se considera que las falsas denuncias de abuso sexual en el contexto del síndrome provocaban en el niño más estrés que el causado por las falsas enfermedades físicas inducidas por las madres. En algunos casos, fueron halladas lesiones físicas provocadas en zonas genitales, que no son encontradas comúnmente en diagnósticos validados de abuso sexual infantil. Los niños habían sido sometidos a múltiples evaluaciones y revisaciones médicas por insistencia de la madre promoviendo su revictimización.

A continuación, se presentan algunos de los indicadores a tener en cuenta al momento de realizar el diagnóstico de síndrome de Münchausen por poderes. Modificado y ampliado de Garrote et al. (2008). 
- Los signos y síntomas referidos por la madre son extraños y el diagnóstico médico no coincide con los hallazgos en las pruebas objetivas.

- Se observa ausencia de mejoría frente a los tratamientos médicos, o inusual intolerancia a los mismos.

- El agresor presenta incongruencias en el relato de los síntomas y no expresa alivio al informársele la mejoría del niño.

- El cuidador insiste en la utilización de procedimientos médicos e internaciones y manifiesta un tenor afectivo que no coincide con la gravedad de los síntomas del niño.

- Los signos y los síntomas se presentan en presencia del cuidador y desaparecen si se lo aleja del niño.

- Se reporta historia de hermanos con enfermedad inusual, o fallecidos en forma inexplicable.

- En ocasiones, el cuidador hace una exposición pública de la enfermedad de su hijo, para obtener un beneficio secundario (e.g., donaciones para pagar supuestos tratamientos).

- Se encuentran antecedentes personales del cuidador acerca de trastornos de somatización, y/o de familiares con enfermedades poco comunes.

- La madre, el padre o el cuidador poseen conocimientos médicos, o desempeñan una profesión afín, en muchas ocasiones tergiversan las indicaciones médicas de tratamientos anteriores.

- Los médicos llegan a dudar de su saber al no poder hacer el diagnóstico o ubicar los síntomas y los signos en entidad nosológica alguna. Se repite un patrón de egresos sin alta médica en internaciones previas.

\section{Protocolo de recolección de datos para los efectores de salud}

El comportamiento parental y lo referido por otros adultos que puedan dar testimonio de la historia personal, familiar y social del niño/a, deben ser valorados con rigurosidad. Los antecedentes médicos, psicológicos y psiquiátricos tanto de los hermanos, como de los padres, las posibles intervenciones legales en la familia y toda documentación que acredite la historia relatada por el cuidador, son también aspectos relevantes a evaluar.

Es imprescindible realizar una exhaustiva revisión de las historias clínicas previas y recopilar los antecedentes de las intervenciones de los servicios de protección y promoción de derechos de los niños, niñas y adolescentes, de los juzgados, de las 
instituciones recreativas y escolares. Para tal fin, se presenta a continuación una tabla integradora de los datos necesarios a relevar por los efectores de salud, la cual podrá ser la base de un eventual protocolo para ser utilizado en el abordaje de esta patología que pone en riesgo la vida de los niños, niñas y adolescentes.

Tabla 2. Modelo de registro de datos para ser relevados por los efectores de salud

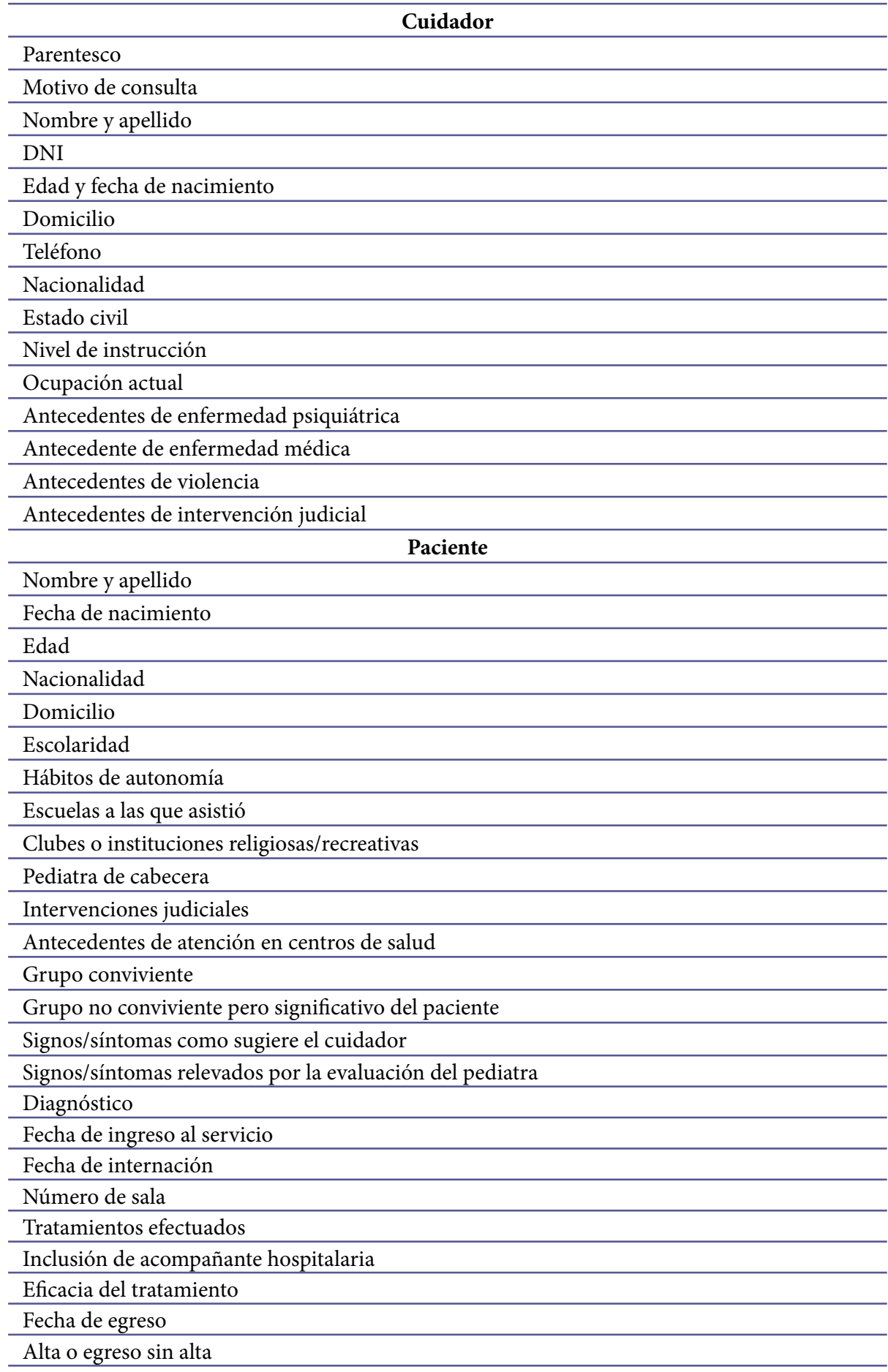




\section{Registro epidemiológico efectuado en la unidad de violencia familiar del hospital general de niños Pedro de Elizalde, durante el período 2001-2017}

Debido al progresivo incremento de casos se subdivide el relevamiento en dos períodos para evaluar posteriormente este aumento con otras variables intervinientes. En el período comprendido entre los años 2001 y 2009, ingresaron once casos con sospecha de este síndrome y en el comprendido entre 2009 y 2017, 43 casos.

En relación a los diagnósticos confirmados, se registraron siete entre 2001-2009 y diecisiete entre 2009-2017. Dando un total de 54 sospechas y 24 casos confirmados desde el año 2001 al 2017.

Con referencia a las edades de los niños y niñas se establecieron los siguientes rangos. La proporción entre varones y mujeres víctimas de este síndrome fue de 14 mujeres y diez varones.

En cuanto al vínculo del inductor de la enfermedad con el niño o la niña, la relación fue: 23 madres y un padre.

En relación con los antecedentes familiares, de los 24 casos confirmados, se reportó que doce tenían antecedentes de hermanos que presentaban patologías médicas múltiples, fallecidos, con SMPP, institucionalizados y con maltrato físico de alto nivel de riesgo. En la muestra se registró también el fallecimiento de un primo conviviente al cuidado de la madre inductora de la patología de su hijo.

En cuanto al alta, se observó que, de los 24 casos, doce fueron alojados en instituciones fuera del control del agresor, cuatro con familiares en su mayoría abuelas, uno con el padre, uno con la madre en seguimiento ambulatorio y seis egresos con la madre sin alta otorgada por el hospital.

\section{Estrategias para el diagnóstico}

El diagnóstico del síndrome de Münchausen por poderes es particularmente dificultoso y se sugiere que, ante la duda, la primera medida a implementar sea la organización del resguardo del niño, niña o adolescente. En el caso de existir

Tabla 3. Registro epidemiológico. Unidad de Violencia Familiar, Hospital Gral. de Niños Pedro de Elizalde. Número de casos según rango etario

\begin{tabular}{lr}
\hline \multicolumn{1}{c}{ Rango etario } & Número de casos \\
\hline 0-3 años & $12(50 \%)$ \\
\hline 3-6 años & $4(16,6 \%)$ \\
\hline 6-9 años & $2(8,4 \%)$ \\
\hline 9-18 años & $6(25 \%)$ \\
\hline
\end{tabular}


un alto grado de sospecha al abordar a un paciente ambulatorio, se lleva a cabo la internación del mismo y se da intervención a los servicios de protección de derechos. Además, se solicita acompañante hospitalario para cuidar del niño o de la niña las 24 horas, explicitando su función en el diagnóstico de esta patología.

Existen dos tipos de diagnósticos: el primero, por inclusión o video vigilancia encubierta, a través del cual se coloca una cámara monitoreada permanentemente que registra los posibles malos tratos del cuidador hacia el niño/a; el segundo, por exclusión, que consiste en el apartamiento de la madre o cuidador para poder evaluar la continuidad o no de la sintomatología en su ausencia.

El diagnóstico por exclusión es considerado más seguro y ético para el niño o la niña que el de inclusión, ya que no lo expone a un posible nuevo maltrato. La video vigilancia encubierta es muy útil como prueba objetiva judicial, siempre y cuando se monitoree en tiempo real para evitar un nuevo daño al paciente. Simultáneamente, es imprescindible realizar la revisión de la historia familiar, social y personal así como de toda la información que aporte datos para confirmar o descartar el diagnóstico presuntivo de síndrome de Münchausen por poderes.

El arribo al diagnóstico precoz se lleva a cabo a partir del trabajo interdisciplinario entre los psicólogos especialistas en maltrato infanto-juvenil, los médicos pediatras y los trabajadores sociales. Radica en evitar la progresión de la sintomatología y la ejecución de métodos invasivos de diagnóstico, que pueden provocar la muerte del paciente. La comunicación efectiva entre los diferentes efectores de salud favorecerá una correcta evaluación de este síndrome.

Según Indart (2018) existe un retraso de trece meses entre el momento en que los niños y las niñas presentan los síntomas iniciales, hasta que se efectúa el diagnóstico de síndrome de Münchausen por poderes.

En los estudios reportados por Flaherty y MacMillan (2013), un 30\% de los niños y las niñas víctimas de esta patología, tienen una enfermedad médica subyacente y la mayoría presentan signos iatrogénicos a partir de las múltiples intervenciones que se les realizan.

\section{Diagnóstico de púberes y adolescentes durante la internación}

Es factible comprobar que los púberes o los adolescentes comienzan a participar de este tipo de maltrato y en ocasiones llegan a la adultez como portadores del síndrome y lo ejercen sobre otros. A diferencia de los niños pequeños, que según las estadísticas registran la mayor parte de los casos detectados, en púberes y en adolescentes el síntoma fabricado por el adulto es tomado como propio, sostenido y a veces auto-provocado. En estos casos, el relato del púber coincide con el discurso del cuidador en relación con el historial de enfermedades y con el síntoma actual.

Puede observarse un doble circuito de violencia, por un lado, física y psicológica generada por el cuidador hacia el niño o la niña, y por el otro, física y emocional ejercida por ellos sobre su propio cuerpo, como forma de responder a la demanda por parte del adulto. 
De esta manera, se establecen vínculos parento-filiales construidos sobre una falsa connotación positiva del dolor físico y de la enfermedad. Este padecimiento puede ser para el niño o niña una promesa de curación o una forma de sostener el vínculo, ya que al complacer al perpetrador intenta evitar el abandono. La sintomatología puede presentarse en la mente del paciente como una señal para el interés médico, lo que podría darle una de las pocas oportunidades de ser visto y, en el caso de ser así, recibir protección. Existe un fantasma crónico de enfermedad que recae en el cuerpo del niño o niña, lo que constituye una amenaza constante.

Luego de la separación del progenitor, aparece la idea de muerte en primer plano que es expresada en forma descarnada por los pacientes. En los casos donde existe enfermedad orgánica inducida por cuidadores, siempre hay daño emocional. En algunos pacientes la presencia de angustia, ansiedad, delirios y pasajes al acto también responden a la inducción. Del mismo modo, la presencia de dicha sintomatología puede llevar a la persuasión del perpetrador para que se efectúe una internación psiquiátrica.

Cuando el dolor es prevalentemente psíquico, es mucho más difícil de trabajar en forma interdisciplinaria dentro del ámbito hospitalario y con las otras instituciones, debido a la ausencia de evidencia médica en relación con la sintomatología. El daño para el niño o la niña es igualmente devastador, sin embargo, el maltrato emocional es una categoría que permanece devaluada y subdiagnosticada.

\section{Descripción fenomenológica de la conducta previa y posterior a la develación} del síndrome.

Previo a la separación del cuidador:

a. El síntoma es inducido y producido en el cuerpo-mente del niño o de la niña.

b. El paciente repite la historia construida por el adulto con la creación de un enemigo externo peligroso (e.g., padre excluido). El rol actuado por la madre es de salvadora, a su vez amada y temida, mediante el proceso activo de aislamiento familiar.

c. Se evidencia una diferencia entre la verdad narrativa y la histórica, la primera es la forma de adaptación y reproducción del discurso materno que le permite al niño o a la niña sobrevivir. La verdad histórica será recuperada parcialmente en una segunda etapa, cuando el paciente pueda evocar los hechos de la realidad vividos y silenciados por el adulto.

d. El paciente oscila entre hablar y callar, siendo la primera una conducta peligrosa por la respuesta materna. El hecho de callar, le genera una intensa angustia que conlleva un esfuerzo emocional por el temor al abandono materno. Una de las consecuencias de los traumas repetidos es el aislamiento y el silenciamiento de la palabra.

e. En ocasiones el paciente queda sometido a las presiones intencionales del perpetrador o grupo familiar y a las involuntarias del equipo tratante. 
f. El vínculo psicoterapéutico promueve en la vivencia actual del niño o de la niña un espacio protector, mientras no estén cuestionados los dichos de la madre. Los pacientes aportan datos de familiares significativos de su primera infancia, a pesar del esfuerzo materno por impedirlo. En ocasiones, parte de la historia vivida emerge escapando al control del adulto.

Posterior a la separación del cuidador:

a. Intensa angustia y aparición de ideas de muerte.

b. Sostén y resistencia en cuanto a la idea de enfermedad que organizaba su vida.

c. Disminución paulatina de la ansiedad en relación con el alejamiento del perpetrador.

d. Ambivalencia afectiva con respecto al terapeuta y al equipo interviniente. Manifestaciones de ira, llanto y también demostraciones de cariño y demanda de afecto.

e. Hermetismo con relación a los síntomas fabricados por su perpetrador. Se refiere a ellos de forma tangencial o en actividades cotidianas no relacionadas con los mismos.

f. Reconstrucción de su historia con recuerdos que se reformulan en cuanto al tenor afectivo que los niños o las niñas les adjudican. Reorganización de la memoria a partir de la aparición de nuevas evocaciones amables vedadas por sus madres, con relación a familiares significativos.

\section{Comportamiento de la madre perpetradora y trastornos psiquiátricos asociados}

Las madres perpetradoras de este síndrome se caracterizan por mantener una actitud destructiva ante el estrés, violar conscientemente las normas, ser muy hábiles para el engaño y proclives a mentir en relación con detalles irrelevantes. Utilizan en forma preponderante el mecanismo de defensa de la disociación, aunque no presentan en general un cuadro compatible con una estructura psicótica. Se observa que sus acciones están regidas por un proceso de pensamiento de tipo perverso: simulan ser buenas madres, al mismo tiempo que maltratan a sus hijos. A pesar de tener conocimientos con relación a cuestiones médicas, van dejando pistas de su accionar que finalmente terminan delatándolas ante los profesionales que asisten al paciente (Feldman, 1999, como se citó en Rodriguez Cely, Gomez Rativa y Mesa Bayona, 2003).

Los comportamientos que las caracterizan, según Trejo Hernández et al. (2006), son la complacencia, el apoyo al personal médico, así como la cooperatividad y la permanencia constante en el hospital. A pesar del maltrato ejercido se manifiestan con predisposición ante las demandas de sus hijos y con una actitud sobreprotectora. Evidencian satisfacción al referirse a las enfermedades del paciente a través de 
expresar sentimientos de heroísmo por el cuidado dispensado por ellas hacia sus hijos y en ocasiones a otros niños. Estas madres tienden a la búsqueda de un lugar protagónico dentro de la institución que asiste a su hijo, extendiéndose también hacia la comunidad en la que habitan. En algunos casos, se ha reportado la adquisición de beneficios económicos a partir de la inducción de la enfermedad y de la promoción de la misma en su medio social.

En general, estas madres no poseen una relación de pareja estable y aíslan al paciente del resto de la familia, ya sea nuclear o extensa de ambas ramas. Las que conviven con el padre del niño, presentan conflictos vinculares o tienen una relación en la cual someten a su pareja.

En cuanto a las características de personalidad, es común que presenten ideación suicida y amenazas de suicidio posteriores al diagnóstico y a la separación del niño. Sobre la existencia de patología asociada, suelen presentar consumo problemático de alcohol y de sustancias psicoactivas.

Las madres perpetradoras de este síndrome evidencian al momento de la evaluación diagnóstica un trastorno histriónico, límite, narcisista o no especificado de la personalidad. Solo en muy raras ocasiones la madre tiene un trastorno psicótico. En este último caso, el manejo de la evaluación se complejiza debido a la preponderancia de la sintomatología psicótica por sobre el diagnóstico de síndrome de Münchausen por poderes. Estas madres suelen referir una historia de maltrato significativo durante su infancia, de abuso sexual y maltrato físico.

No existe evidencia que sugiera que la perpetradora de este tipo de maltrato infantil sea inconsciente del daño que inflige al niño, puesto que la planificación y la organización implican hasta la más aguda atención y la mantención del secreto de sus actos. Pueden ser peligrosas con otras personas, dañar a un familiar o niño a su cargo y también llegan a serlo con el personal de la salud. La ejecución del síndrome de Münchausen por poderes es consciente, voluntaria y violenta.

\section{Contratransferencia del equipo tratante}

Los profesionales que intervienen en el abordaje del síndrome de Münchausen por poderes, experimentan sentimientos de protección para con el paciente, en la necesidad de sostener el vínculo. Asimismo, reciben los embates de la ambivalencia del niño, niña o adolescente, que ha vivido una realidad mortífera construida por su figura materna, quien a pesar de las consecuencias letales de su accionar sigue proporcionándole identidad.

El equipo de salud tratante es sometido también a los efectos nocivos de la manipulación del perpetrador. Este último, por lo general amenaza, hostiga y amedrenta a través de conductas querellantes y violentas. En ocasiones, acosan a los profesionales que asisten al paciente obligándolos a tomar medidas de protección personales, lo que les genera temor y sensaciones persecutorias. 


\section{Egreso del centro de salud}

Al momento del egreso, debido a que el perpetrador transgrede las normas y la ley, será necesario propiciar acciones de cuidado y contención para el niño o la niña frente a situaciones intimidantes y angustiosas. Además, se requerirá la comunicación y puesta en común de estrategias de egreso del hospital con los actores intervinientes, intra e interinstitucionales.

En pocas ocasiones, existe familia ampliada que pueda cuidar del paciente, en estos casos la evolución de los niños o niñas tiende a ser más favorable. Algunas veces, se producen egresos sin alta planeados por la madre, en otros casos el alta se efectúa a través de la implementación de recursos institucionales, como el ingreso a hogares terapéuticos o convivenciales.

\section{Tratamiento psicológico}

Durante el proceso de develación, el niño comienza a redescubrir su subjetividad, reconoce un nuevo cuerpo que cada día padece menos y con el que cuenta para enfrentar una nueva vida llena de posibilidades.

Las emociones son dicotómicas, amor y odio lo separan y lo unen al mismo tiempo de los profesionales que intentan asistirlo. Continúa sintiendo miedo y preservando el síntoma que le dio identidad hasta ese momento. Luego de un tiempo, cuando el distrés deja lugar a cierto equilibrio, comienza a construir nuevos vínculos.

Durante el tratamiento, el niño o la niña tiene el gran desafío de empezar a creer en otro adulto que no sea su madre, y el psicólogo, el de buscar dentro de la historia personal del paciente algún recurso resiliente que le permita construir nuevas configuraciones.

Si cuenta con otros referentes familiares o miembros del entorno social y asistencial que lo contiene, se comienza a formar una nueva red, en donde el niño o la niña puede encontrar respuestas a lo vivido. Estos pacientes habitualmente son aislados por su madre, sin ofrecerle la posibilidad del encuentro con otros significativos.

A veces, el paciente está preparado psíquicamente para verbalizar y otras, solo el juego o el respetuoso silencio van afianzando los primeros pasos en la relación terapéutica. Algunas características personales lo ayudan a transitar este camino. Schwalb (2012) escribió que los sobrevivientes de la guerra, muchas veces silencian su historia. Sus ojos vieron el horror y las palabras parecen no poder abarcar la dimensión de lo vivido. La herencia resiliente, en estos casos, no tiene que ver con lo dicho sino con un silencio colmado de significación.

El niño cuenta con una red y una historia que no solo se remontan a su familia nuclear, otros protagonistas pueden haber marcado una huella que en algún momento se resignificará con nuevos modelos de identificación. En esta primera etapa, el paciente determinará cuándo y cómo surgirán las preguntas y respuestas que le ayuden a comprender lo ocurrido, que justifiquen que la persona que supuestamente debía cuidarlo y protegerlo, lo indujo a la enfermedad y muchas veces lo acercó a la muerte. 
$\mathrm{Al}$ abordar el tratamiento, se organizan las sesiones en encuentros alternados con el niño, su referente adulto actual, hermanos u otros significativos. Se planifican sesiones individuales y vinculares, para hacer foco en la comunicación del sistema cuyos componentes -paciente y demás integrantes-interactúan entre sí y a su vez con el medio sociocultural del que forman parte.

En general, si se cuenta con el progenitor que no maltrata u otro adulto que pueda colaborar, se lo incluye en algunas entrevistas y/o sesiones vinculares. Es muy productivo realizar una sesión vincular entre el paciente y el actual cuidador para trabajar sobre una escena de conflicto entre ambos manteniendo la alianza terapéutica. La confianza en el otro, en casos de pacientes con este tipo de maltrato, está mellada por la experiencia. La desconfianza, en ocasiones, puede interferir en la constitución de otros vínculos.

El perpetrador de este síndrome, en su gran mayoría las madres, tiene una muy escasa adherencia al tratamiento, ya sea psicológico o psiquiátrico. Esta característica, imprime un factor de alto riesgo, anula la posibilidad de prevención de la patología y tiende a alejar la posibilidad de que el niño se revincule con su madre o que ella participe en el tratamiento de su hijo.

Algunas de las metas terapéuticas serán que el paciente logre visualizar y aceptar lo que le sucedió, a través de distanciarse de lo ocurrido y de ser una persona diferenciarse de su madre. Se tenderá a que logre tomar conciencia de la realidad que lo rodea y que pueda atribuir la responsabilidad de lo sucedido al adulto perpetrador. Otro de los objetivos será que el niño pueda expresar el afecto bloqueado que quedó ligado a escenas o pensamientos traumáticos. Al mismo tiempo, se promoverá a través del uso del pensamiento crítico, que pueda adquirir confianza en otros que lo acompañen en su crecimiento y cuiden de él sin manipulaciones. No es la función del terapeuta juzgar a quien fue su figura de apego. En el momento oportuno se hará referencia a la imposibilidad de su cuidado y a las aparentes causas del maltrato, según la etapa de desarrollo psíquico del niño, niña o adolescente.

\section{Conclusiones}

La problemática del síndrome de Münchausen por poderes se extiende a los distintos efectores de salud a la hora de detectar, diagnosticar y brindar tratamiento al niño, niña o adolescente y al perpetrador. Esta situación es potencializadora de la búsqueda de herramientas que permitan visualizar la temática. A tal fin, se concluye con una revisión de los aspectos más importantes a tener en cuenta para el abordaje integral de esta patología.

a. La compleja tarea de los médicos pediatras para arribar al diagnóstico, quienes son sometidos al engaño de los responsables del maltrato. En ocasiones, este síndrome coexiste con patología real en algunos niños o niñas, tales como leucemia, enfermedades reumáticas, diabetes, entre otras, lo que genera confusión y conflicto en los médicos tratantes de las distintas especialidades. 
Es necesaria la articulación con otras disciplinas, como la psicología y el trabajo social, a la hora de realizar el diagnóstico, la exclusión de la madre, el tratamiento y el seguimiento del paciente.

b. La desmitificación de la creencia social y cultural sobre la capacidad innata de todas las madres para cuidar de sus hijos. Los datos epidemiológicos relevados en el presente trabajo, en el período 2001-2017, de una población de un hospital público de CABA, con 54 casos, arrojó 24 casos confirmados. En cuanto al vínculo del inductor de la enfermedad, los resultados fueron 23 madres, un padre.

c. La experiencia con pacientes víctimas de este tipo de maltrato confirma que, en algunos casos, cuando los niños y las niñas llegan a púberes o adolescentes comienzan a participar de este tipo de patología y arriban a la adultez siendo ellos portadores del síndrome y pudiendo ejercerlo sobre otros.

d. El obstáculo que se presenta con los servicios de protección de derechos de los niños, niñas y adolescentes, quienes en ocasiones no poseen el conocimiento en la temática referida, ni los recursos, ni la celeridad para dar una respuesta adecuada a las necesidades de resguardo.

e. La minimización por parte de los organismos de la justicia de la intencionalidad del daño psíquico y físico provocado por el agresor, lo que pone en riesgo la vida de los pacientes. Como consecuencia de esta desestimación no consideran prioritaria la realización de un tratamiento psicológico y/o psiquiátrico por parte de la madre, debidamente monitoreado a través de un seguimiento exhaustivo.

f. La indagación de datos ocultos por la madre, la exhaustiva revisión de las historias clínicas previas, el minucioso relevo de las intervenciones de los servicios de protección y promoción de derechos, los juzgados, las instituciones recreativas y escolares, proporcionan un aporte que a veces se ve sesgado por el subregistro de los mismos. La tabla integradora de datos para completar por los efectores de salud que se presenta en este artículo, pretende ser la base de un futuro protocolo que se confeccionará para ser utilizado en el abordaje de esta patología.

g. Los perpetradores de este síndrome, presentan trastornos graves de la personalidad, con casi inexistente adherencia al tratamiento, lo que permite una limitada tarea de prevención.

A partir de este trabajo, con el fin de realizar un aporte al resguardo de niños, niñas y adolescentes, se dará inicio a una investigación sobre la determinación del perfil psicológico del agresor. Para ello, se utilizará una escala de personalidad que permita arrojar datos psicométricos sobre la base de la evidencia, para brindar un tratamiento adecuado a los inductores de esta patología. 


\section{Referencias}

Asociación Americana de Psiquiatría (1994). Manual diagnóstico y estadístico de los trastornos mentales. DSM-4. Masson.

Asociación Americana de Psiquiatría (2002). Manual diagnóstico y estadístico de los transtornos mentales. DSM-4 TR. Masson.

Asociación Americana de Psiquiatría (2014). Manual diagnóstico y estadístico de los trastornos mentales. DSM-5. Arlington.

Del Bagge, P. Lovaglio, G. Perez, R. y Stabilito, L. (26-29 de abril de 2017). Enfermedad inducida por cuidador. Registro epidemiológico 2001-2017. [Mesa redonda]. XXXII Congreso Argentino de Psiquiatría, Mar del Plata, Argentina.

Flaherty, E. y Mac Millan, H. (2013). Caregiverfabricated illness in a child: A manifestation of child maltreatment. Pediatrics, 132, 590-597. https://doi.org/10.1542/peds.2013-20145

Garrote, N. Indart, J. Puentes, A. Smith, M. Del Bagge, P. y Pérez Colombier, M. (2008). Síndrome de Münchausen por poder y manifestaciones de supuesto evento de aparente amenaza a la vida Archivos Argentinos de Pediatría, 106 (1), 47-53.

Glaser, D. y Davis, P. (2019). For debate: Forty years of fabricated or induced illness (FII): Where next for paediatricians? Paper 2: Management of perplexing presentations in-cluding FII. Archives of Disease in Childhood, 104, 7-11. doi:10.1136/ archdischild-2016-311326. HTTPS://WWW. RESEARCHGATE.NET/PUBLICATION/324239235

Indart, J. (2018). Enfermedad inducida o fabricada por cuidadores o síndrome de Münchausen por poderes. En N. Garrote (Eds.), Maltrato infantil. Aportes para su detección, abordaje y prevención (pp. 134-139). Journal.
Jiménez Hernández, J.L. (2015). Síndrome de Münchausen por poderes. Un tipo sutil de niños maltratados. Tesis doctoral inédita. Facultad de Medicina, Universidad Complutense de Madrid. HTTPS://EPRINTS.UCM.ES/54630/1/5331023885.PDF

Meadow, R. (1993). False allegations of abuse and Munchausen syndrome by proxy. Archives Disease Childhood, 68 (4), 444-447. нттр:// DX.DOI.ORG/10.1136/ADC.68.4.444

Meadow, R. (2002). Different interpretation of Munchausen syndrome by proxy. Child Abuse and Neglect, 26, 501-508. HTTPS://DOI. ORG/10.1016/50145-2134(2)00326-5

Rodríguez Cely, L. Gómez Rátiva, M. Mesa Bayona, A. (2003). Estado del arte sobre el síndrome de Münchausen por poderes. Universitas Psychologica 2, (2), 187-198.

Schwalb, V. (2012). Todos somos resilientes. Paidós.

Síndrome de Munchausen por poder. (4 de abril de 2019). Infomed. HTTPS//ARTÍCULOS.SLD.CU/PREVEMI/TAG/ SÍNDROME-DE-MUNCHAUSEN-PORPODER/

Trejo Hernandez, J. Loredo Abdala, A. Orozco Garibay, J. (2011). Munchausen. syndrome by proxy in mexican children: Medical, social, psychological and legal aspects. Revista de Investigación Clínica, 63(3), 253-262. 